Preis (Hrsg.)

Der Arbeitsvertrag 



\section{Der Arbeitsvertrag}

Handbuch der

Vertragsgestaltung

herausgegebenvon

Prof. Dr. Dr. h.c. Ulrich Preis

bearbeitet von

Prof. Dr. Stefan Greiner

Universitätsprofessor, Bonn

Prof. Dr. Dr. h.c. Ulrich Preis

Universitätsprofessor, Köln

Prof. Dr. Christian Rolfs

Universitätsprofessor, Köln

Prof. Dr. Markus Stoffels

Universitätsprofessor, Heidelberg

Dr. Klaus Wagner

Vorsitzender Richter

am Finanzgericht, Düsseldorf

5. Auflage

2015

\section{ottoschmidt}




\section{Zitierempfehlung:}

Preis/Bearbeiter, Der Arbeitsvertrag, I A Rz. 2

und:

Preis/Bearbeiter, Der Arbeitsvertrag, II A 10 Rz. 10 ff.

\section{Bibliografische Information}

der Deutschen Nationalbibliothek

Die Deutsche Nationalbibliothek verzeichnet diese Publikation in der Deutschen Nationalbibliografie; detaillierte bibliografische Daten sind im Internet über http://dnb.d-nb.de abrufbar.

Verlag Dr. Otto Schmidt KG

Gustav-Heinemann-Ufer 58, 50968 Köln

Tel. 02 21/937 38-01, Fax 02 21/93738-943

info@otto-schmidt.de

www.otto-schmidt.de

ISBN 978-3-504-42033-8

(C) 2015 by Verlag Dr. Otto Schmidt KG, Köln

Das Werk einschließlich aller seiner Teile ist urheberrechtlich geschützt. Jede Verwertung, die nicht ausdrücklich vom Urheberrechtsgesetz zugelassen ist, bedarf der vorherigen Zustimmung des Verlages. Das gilt insbesondere für Vervielfältigungen, Bearbeitungen, Übersetzungen, Mikroverfilmungen und die Einspeicherung und Verarbeitung in elektronischen Systemen.

Das verwendete Papier ist aus chlorfrei gebleichten Rohstoffen hergestellt, holz- und säurefrei, alterungsbeständig und umweltfreundlich.

Einbandgestaltung: Jan P. Lichtenford, Mettmann

Satz: Schäper, Bonn

Druck und Verarbeitung: Kösel, Krugzell

Printed in Germany 